Running Header: HEART RATE VARIABILITY IN MIDLIFE

Childhood Parental Warmth and Heart Rate Variability in Midlife:

Implications for Health

Note: Pre-Print Version of Manuscript Currently In-Press at Personal Relationships,

(Uncorrected Proof). 


\title{
Childhood Parental Warmth and Heart Rate Variability in Midlife:
}

\section{Implications for Health}

\author{
Nicholas V. Alen ${ }^{1}$, Richard P. Sloan ${ }^{2}$, Teresa E. Seeman ${ }^{3}$, \& Camelia E. Hostinar ${ }^{1}$ \\ 1. University of California, Davis
}

2. Columbia University

3. University of California, Los Angeles

Acknowledgements: Data used for this research were provided by the longitudinal study titled "Midlife in the United States" (MIDUS), managed by the Institute on Aging, University of Wisconsin, and supported by Grants P01-AG020166 and U19-AG051426 from the National Institute on Aging; and Grants UL1TR001409, UL1TR001881, and 1UL1RR025011 from the NIH National Center for Advancing Translational Sciences (NCATS) Clinical and Translational Science Award (CTSA) program. The authors' efforts on this article were supported by the Dean's Distinguished Graduate Fellowship (DDGF) provided by the University of California, Davis to NVA and NSF grant 1327768 support to CEH. The authors assume full responsibility for this work. The ideas expressed herein do not represent the views of the original collector of the data, the Inter-university Consortium for Political and Social Research (ICPSR), or that of the funding agencies.

Correspondence regarding this article should be addressed to Nicholas V. Alen and Camelia E. Hostinar, Center for Mind and Brain, University of California, Davis, 267 Cousteau Place, Davis, CA 95618. E-mails: nvalen@ucdavis.edu; cehostinar@ucdavis.edu. 


\begin{abstract}
The current study investigated high-frequency heart rate variability (HF-HRV) as a potential mediator between childhood parental warmth and later health and mortality outcomes. Participants were 1,255 adults (56.9\% female). Childhood parental warmth was reported retrospectively at mean age 46; resting HF-HRV was measured at mean age 57; cardiovascular health and self-evaluated health were assessed at mean age 57 and 63, and mortality records extracted at mean age 63 . Results revealed a positive association between childhood parental warmth and resting HF-HRV, as well as associations between higher HF-HRV and reduced risk of having a later cardiovascular health problem and of mortality by age 63 . Mediation analyses revealed a small significant indirect effect of parental warmth, through HF-HRV, on cardiovascular health.
\end{abstract}

Keywords: heart rate variability, parental warmth, cardiovascular, mortality, MIDUS. 
The quality of the social environment during childhood has enduring repercussions for offspring health and wellbeing (Chen, Brody, \& Miller, 2017). In particular, a growing body of research has found associations between characteristics of the parent-child relationship and offspring physiology, with parenting that is high in warmth generally predicting a more moderate and flexible physiological profile (Flannery, Beauchamp, \& Fisher, 2017; Gunnar \& Quevedo, 2007; Repetti, Taylor, \& Seeman, 2002). However, most prior research has focused on links between parenting and the hypothalamic-pituitary-adrenal (HPA) axis, and much less is known about the potential association between parenting and the autonomic nervous system (ANS). Understanding the relations between childhood parental warmth and ANS physiology has important implications for public health promotion, as more moderate and flexible ANS physiology has been associated with several beneficial health outcomes (Thayer, Yamamoto, \& Brosschot, 2010). The present study aimed to (1) address this research gap by testing the association between childhood parental warmth and ANS physiology, indexed through resting high frequency heart rate variability (HF-HRV); (2) add to the growing body of evidence linking HF-HRV to lifelong health and mortality outcomes; and (3) test HF-HRV as a mediator between parental warmth and health and mortality.

\section{Parental Warmth and Health}

Parental warmth refers to parenting that is affectionate, consistent, and marked by sensitive responding to the child's needs (Zhou et al., 2002). Past mediation studies attempting to explain the association between parental warmth and health have typically focused on two mechanisms: (1) health behaviors, whereby warmer parenting is associated with healthier behavior patterns -e.g., diet, physical activity (Davids, Roman, \& Leach, 2017; Graves, Wang, Mead, Johnson, \& Klag, 1998); and (2) stress physiology and the immune system, such that 
warmer parenting predicts more moderate stress reactivity and more adaptive inflammatory processes, which in turn are associated with better health outcomes (Chen, Miller, Kobor, \& Cole, 2011; Chen et al., 2017; Miller \& Chen, 2010; Uchino \& Way, 2017). However, most studies investigating the link between parental warmth and biological processes have focused on the HPA axis and the immune system, and the relatively fewer studies on parental warmth and the ANS are largely limited to youth, precluding the testing of ANS physiology as a mediator between parental warmth and long term health outcomes, such as cardiovascular disease and mortality.

\section{Parental Warmth and Offspring Physiology}

Research with children and adolescents has shown that warm parenting can dampen neuroendocrine stress responses (Hostinar, Sullivan, \& Gunnar, 2014) and attenuate the effects of adversity on youth physical health (Chen et al., 2017; Farrell, Simpson, Carlson, Englund, \& Sung, 2016). Warmer parenting may therefore help dampen the offspring's general physiological reactivity during early-life, helping to maintain the offspring's physiology within more moderate set points, potentially "programming" the development of physiological profiles that are more moderate and adaptive (Miller, Chen, \& Parker, 2011).

Parenting may also influence the development of offspring physiology by shaping the development of emotion regulation. During early life, parents act as primary regulators of their offspring's affect and physiology (Morris, Silk, Steinberg, Myers, \& Robinson, 2007). Because of this, parents who sensitively respond when their child is in distress, providing scaffolding assistance to the child's attempt to regulate their own physiological reaction, help create internalized patterns of regulatory processes (Thompson, 1994), potentially leading to the development of more moderate and well-regulated ANS physiology. While most studies 
investigating early life "programming" of offspring biology have focused on the HPA axis, there is growing evidence supporting the need to better understand how early life environmental characteristics, particularly parenting, may predict the development of ANS physiology (Propper \& Moore, 2006).

\section{Heart Rate Variability}

A common technique for investigating individual differences in ANS physiology is to measure heart rate variability (HRV) (Laborde, Mosley, \& Thayer, 2017). HRV is a measurement of the beat-to-beat changes in heart rate, and high frequency HRV (HF-HRV) is often used as an index of parasympathetic influence, via the vagus nerve, over cardiovascular activity (Porges, 2007). High resting HF-HRV is believed to represent ANS physiology that can flexibly adapt to changing environmental demands, increasing heart rate during times of threat, then quickly returning to a calm resting state once the threat has subsided (Appelhans, \& Luecken, 2006).

Previous research on the association between parenting characteristics and resting HFHRV is somewhat limited. Nevertheless, the evidence so far suggests an association between positive parenting characteristics and high resting HF-HRV. For example, infants of mothers who warmly responded to their infant's communication cues had higher resting HF-HRV (Porter, 2003). In addition warmer parenting (e.g., high involvement, high support) has been shown to predict higher resting HF-HRV in adolescents (Graham, Scott, \& Weems, 2017), and reduced decreases in resting HF-HRV over a one-year period (Fox, Aldrich, Ahles, \& Mezulis, 2018). Experimental evidence from randomized controlled trials has also revealed associations between (1) increased sensitivity following parenting intervention and higher resting HF-HRV in preschoolers (Bell, Shader, Webster-Stratton, Reid, \& Beauchaine, 2018), and (2) reductions in 
negative parenting following intervention and higher resting HF-HRV in 9-year olds (Tabachnik, Raby, Goldstein, Zajac, \& Dozier, 2019).

There is also evidence of associations between parenting quality and HRV change during challenge, sometimes referred to as vagal withdrawal, though findings have been mixed, with warmer or more sensitive parenting sometimes predicting increased HRV change (Calkins, Graziano, Berdan, Keane, \& Degnan, 2008; Perry et al., 2012), and sometimes predicting decreased HRV change (Hastings et al., 2018). While these mixed results highlight the complexity of HRV change research, it suggests that parenting quality may influence child statelevel ANS physiology in addition to the trait-level ANS physiology captured by resting measures.

\section{HF-HRV and Health}

High resting HF-HRV has been previously associated with better physical health and reduced mortality risk. Low resting HF-HRV has been linked to hypertension (Singh et al., 1998), high cholesterol (Christensen, Toft, Christensen, \& Schmidt, 1999), and coronary heart disease (Liao, Carnethon, Evans, Cascio, \& Heiss, 2002). In addition, low resting HF-HRV has also been previously associated with increased risk for diabetes (Liao et al., 1995) and all-cause mortality (Gerritsen et al., 2001; Tsuji et al., 1994). However, some studies have failed to find associations between HRV and cardiovascular risk factors (Klutting, Kuss, \& Greiser, 2010). Given this mixed evidence, it is important to further test the relation between HRV and health outcomes using large samples.

\section{Current Study}

The present study aimed to (1) expand our current understanding of the association between childhood parental warmth and adult ANS physiology, (2) investigate the relations 
between ANS physiology and long-term health and mortality outcomes in older adulthood, and (3) test HF-HRV as a potential mediator of the relation between parental warmth and health and mortality. Considering some of the previously reported associations between warm parenting and high resting HRV, and high resting HRV and better health and reduced mortality risk, we developed three hypotheses: (1) we hypothesized that adults who reported retrospectively to have received warmer parenting during childhood would exhibit higher resting HF-HRV in midlife; (2) we hypothesized that higher resting HF-HRV would predict better physical health outcomes (cardiovascular health, self-evaluated health) and reduced risk of mortality, and (3) we hypothesized that resting HF-HRV would mediate the relation between childhood parental warmth and health and mortality outcomes in older adulthood.

\section{Methods}

\section{Participants}

Data for this study were drawn from the Midlife Development in the United States (MIDUS) longitudinal study. The first MIDUS wave involved phone and mail surveys to a nationally representative sample of adults $(N=7,108)$. In 2004-2009, a subset of this sample $(n=$ 1,255) were recruited for a follow up study, the Biomarker Project of MIDUS 2, involving assessments of biological markers. The third wave, MIDUS 3 began in 2013, and involved phone survey assessments similar to the first wave. Complete details on the MIDUS study are available at www.midus.wisc.edu.

Participants in the current study include individuals who participated in all three MIDUS waves. Of the 1,255 participants who took part in the MIDUS 2 Biomarker Project, 1,148 had technically acceptable resting HF-HRV data. Of the individuals who returned for MIDUS 3, 938 had data on cardiovascular health, and 943 had data on self-evaluated health. Participants 
included in the current study were: middle-aged, with a mean age at MIDUS 2 of 57.3 (range = 35 to 86 ); predominantly Caucasian (78.7\%), though a large subset of the sample were African American (17.2\%), 1.4\% were Native American, 0.2\% Asian, and 2.5\% Other (please see Table 1 for complete sample demographics). On average, participants had moderate childhood socioeconomic status (SES), with $41.3 \%$ of participants reporting that at least one of their parents completed some college. Just over half of participants were female (56.8\% female; $43.2 \%$ male).

\section{Measures}

Childhood Parental Warmth. Childhood parental warmth was measured retrospectively at MIDUS 1 (mean age 46) using a validated questionnaire (Rossi, 2001). Participants were asked to reflect on their childhood experiences, and answer seven questions regarding the quality of their relationship with each parent (participants completed separate 7-item scales for their mother and their father). Questions assessed how much that parent understood the participant's worries; how much attention, effort, and affection that parent provided; as well as the participant's subjective interpretation of the overall quality of the relationship (e.g., "How would you rate your relationship with your mother/father during the years you were growing up?"). Responses were coded on a 4-point Likert-type scale, ranging from "Not at all" to "A lot"; or, regarding quality of the relationship, from "Poor" to "Excellent"; such that higher scores reflected more parental warmth. Both maternal and paternal warmth scales showed high internal consistency (Cronbach's alpha of .91 and .92, respectively). The maternal and paternal scores were averaged together to provide a measure of combined parental warmth.

High-Frequency Heart Rate Variability. High-frequency heart rate variability (HFHRV) was measured during MIDUS 2, using a 3-lead electrocardiogram (ECG) attached to the chest in Einthoven's triangle configuration. ECG recordings took place in the morning, after an 
overnight clinic stay, following breakfast, with no caffeine consumption permitted. ECG data were recorded during an 11-minute seated, resting baseline period, as part of a larger ECG protocol involving cognitive tasks. The current study utilized data from the resting baseline measure. ECG data were digitized at $500 \mathrm{~Hz}$ by a 16-bit National Instruments analog-to-digital board. ECG wave R peaks were visually inspected and cleaned. Arrhythmias were handled using interpolation. Interbeat-intervals (IBIs) were processed through a Fast Fourier transformation algorithm, with a high frequency band filter set at $0.15-0.50 \mathrm{~Hz}$, providing mean HF-HRV from two 300-second epochs, which were then averaged together. If uncleanable noise precluded the collection of a minimum of 180 continuous seconds, data from that participant were not calculated.

Cardiovascular Health. To create a measure of cardiovascular health, we used participants' answers to ten questions, during MIDUS 3 (mean age 63), regarding the presence or absence of heart problems as diagnosed by a physician. Participants were asked if they had ever been diagnosed by a physician with any of the following: stroke, heart attack, high blood pressure, valve disease, blocked artery, irregular heartbeat, heart murmur, or heart failure; participants were also asked if they had ever had a major heart procedure, and if they currently experience chest pain while walking. Participant responses to these questions were used to create a dichotomous variable, such that individuals who answered "No" to all questions were coded as “0" (excellent cardiovascular health), and individuals who answered "Yes" to at least one question were coded as " 1 ". We also created a count variable by summing the total number of "Yes" responses, such that higher scores represented more cardiovascular health problems.

Self-Evaluated Health. Self-evaluated health was measured during MIDUS 3 via phone surveys. This measure was indexed using one item, answered on a 5-point Likert-type scale: "In 
general, would you say your physical health is excellent, very good, good, fair, or poor?" Responses to this question were reverse coded from the original coding scheme so that higher scores indicated better self-evaluated health (e.g. "poor" $=0$; "excellent" $=4$ ). This question has been found in previous studies to be predictive of objective health outcomes such as mortality (Benjamins, Hummer, Eberstein, \& Nam, 2004).

Mortality. Mortality data were collected through October 2015, using three methods: (1) tracing conducted by the University of Wisconsin Survey Center, which used a variety of database searches to confirm participant identity and status, (2) formal searches through the National Death Index (NDI), and (3) longitudinal sample maintenance procedures, including regular participant outreach.

Covariates. Additional demographic and health data were collected during MIDUS 2, when the ECG protocol was conducted. Demographic information included: age, sex, race, and childhood SES. Race was entered as binary code $($ Caucasian $=0$, Non-Caucasian $=1)$ given that other racial categories had very low frequencies and did not allow sufficient statistical power for inclusion. Childhood SES was indexed through parental education level, as an ordinal variable with 12 categories ranging from "Some primary school" $=1$ to "Doctoral or professional degree" $=12$, in line with prior publications from MIDUS (Fuller-Rower, Curtis, Chae, \& Ryff, 2018). When parents had different education levels, the highest education level between parents was used. Health information included: binary coded exercise habits (engages in regular exercise $=1$ ), binary coded smoking status (current smoker $=1$ ), and whether or not the participant was taking medication that may influence HF-HRV. Medication regimen was coded into two dummy variables representing whether or not the participant was currently taking (1) medications known or believed to increase HF-HRV (parasympathomimetic agents, beta- 
blockers), and (2) medications known or believed to decrease HF-HRV (anticholinergic agents, anti-depressants, sedatives, anti-arrhythmic agents, cardiac drugs, antipsychotics), a method previously used in research on HF-HRV with the MIDUS cohort (Sloan et al., 2017). Menopause status, collected during MIDUS 2, was dummy coded such that both females who had yet to go through menopause and males were assigned a "0", and females who had gone through menopause were assigned a "1", in line with previous MIDUS research on HF-HRV (Sloan et al., 2017). Health data (cardiovascular health, self-evaluated health) were also collected during MIDUS 2 and included as covariates in analyses predicting respective MIDUS 3 health outcomes.

\section{Data Analysis}

Bivariate correlations and regression analyses were calculated using IBM SPSS Statistics version 25, and mediation analyses were performed using structural equation modeling in Mplus version 6.12. To address Aim 1 examining the relation between childhood parental warmth and HF-HRV, a multiple linear regression was performed. Linear regression analysis was performed in 2 steps: first, resting HF-HRV was regressed on parental warmth, then demographic and health related covariates (age, sex, race, childhood SES, exercise, smoking, medication regimen, menopause status) were added to the model. HF-HRV data were positively skewed, so in accordance with standard methods (Malik, 1996), we performed a natural log transformation prior to analyses. Due to the well documented correlation between HF-HRV and heart rate (HR), we followed recent recommendations (de Geus, Gianaros, Brindle, Jennings, \& Berntson, 2018) and calculated HF-HRV adjusted for concurrent HR (aHF-HRV), using the following equation: aHF-HRV $=100 *\left(H F-H R V /(I B I)^{2}\right)$. Initial analyses were performed using unadjusted HF-HRV; post hoc sensitivity analyses were performed for all analyses using aHF-HRV. Positively skewed 
aHF-HRV data were log transformed prior to analyses. Participants excluded from regression analysis due to missing one or more variables were on average 6.3 years older, thus we adjusted for age statistically in all analyses. Participants with missing data did not significantly differ from those included in regards to any other variable. Sample size varies across analyses depending on data availability. In order to correct for multiple comparisons we utilized the Benjamini-Hochberg False Discovery Rate (FDR). With this method an estimate is significant if the p-value is smaller than the corresponding FDR q-value.

To address Aim 2, testing the relation between resting HF-HRV and health and mortality outcomes, four separate regression models were performed. The association between HF-HRV and cardiovascular health was investigated through two models: (1) a binary logistic regression predicting the dichotomous cardiovascular health problem variable, and (2) a generalized linear model with a Poisson distribution predicting the total number of cardiovascular health problems. A Poisson distribution allows for better modeling of the distribution of count data, which is characterized by the absence of values below zero and a positive skew (Atkins \& Gallop, 2007). To test the association between HF-HRV and self-evaluated health, we performed a multiple linear regression with resting HF-HRV as a predictor of self-evaluated health. Finally, to test the association between HF-HRV and mortality we performed a binary logistic regression. All models, with the exception of the model predicting mortality, were run in three steps: (1) first without covariates, then (2) controlling for respective health measured during MIDUS 2, then (3) with demographic and health covariates (age, sex, race, childhood SES, exercise, smoking, medication regimen, menopause status) added to the model. The model predicting mortality was tested in two steps: (1) first without covariates, then (2) with demographic and health covariates. 
To address Aim 3, testing HF-HRV as a mediator between childhood parental warmth and later health and mortality outcomes, we performed four mediation analyses using biascorrected bootstrapping methods (Hayes, 2009), with 1000 samples, in Mplus software. With this method an indirect effect is significant if the $95 \%$ bootstrapped confidence interval does not span zero. In order to address missing data in our mediation analysis, Full Information Maximum Likelihood (FIML) was used. Mplus FIML employs Ecker-Huber-White estimation, which accounts for non-normality and the non-independence of data clustered within families, a method previously used with this data set (Donoho, Seeman, Sloan, \& Crimmins, 2015; Wiley, Gruenewald, Karlamangla, \& Seeman, 2016).

\section{Results}

\section{Preliminary Analysis}

Table 1 provides characteristics of participants included in the analysis. We highlight some of the significant correlations (for complete bivariate correlational results, please see Table 2; note that Spearman rank correlations were used for this correlation table given that some of our outcome variables were skewed). Parental warmth was positively correlated with HF-HRV $\left(r_{s}=.06, p=.04\right)$, such that warmer parenting predicted higher HF-HRV. Higher HF-HRV was associated with better cardiovascular health and reduced mortality risk: HF-HRV was negatively correlated with having a cardiovascular health problem $\left(r_{s}=-.13, p<.001\right)$, total number of cardiovascular health problems $\left(r_{s}=-.13, p<.001\right)$, and mortality $\left(r_{s}=-.09, p=.002\right)$.

Cardiovascular health and self-evaluated health were moderately correlated $\left(r_{s}=-.32, p<.001\right)$, suggesting that these two measures represent different aspects of physical health outcomes. Mortality was negatively correlated with self-evaluated health $\left(r_{s}=-.08, p=.02\right)$.

\section{The Relation Between Childhood Parental Warmth and HF-HRV}


Results from a multiple linear regression revealed a significant positive relation between childhood parental warmth and HF-HRV at the univariate level, $\beta=.06, p=.04$. Results from the final model, presented in Table 3, which included covariates, also revealed a significant positive association between childhood parental warmth and HF-HRV, $\beta=.07, p=.01(F D R q$ $=.025)$. Raw data are presented in Supplemental Figure S1.

\section{HF-HRV and Cardiovascular Health}

To test the relation between HF-HRV and cardiovascular health we conducted two models. First, using a dichotomous dependent variable representing presence or absence of any cardiovascular health problems, we conducted a binary logistic regression. Results revealed a significant association between HF-HRV and cardiovascular health both at the univariate level, $B$ $=-.24, S E=.06, p<.001, O R=.79,95 \% \mathrm{CI}[0.71,0.89]$, and after including demographic covariates, $B=-.14, p=.02, O R=.87,95 \% \mathrm{CI}[0.77,0.98]$. The final model, including covariates related to HF -HRV, again revealed a significant association between HF-HRV and cardiovascular health, $B=-.17, S E=.06, p=.007(F D R q=.0125), O R=.84,95 \%$ CI $[0.74$, 0.95], such that greater HF-HRV predicted lower odds of having a cardiovascular health problem at mean age 63. Raw data for this association are presented in Supplemental Figure S2. In order to test the robustness of this relation we also conducted a generalized linear model (GLM) to see if HF-HRV could predict total number of cardiovascular health problems. Results from this model again revealed a significant association between HF-HRV and total number of cardiovascular health problems (please see Table 4 for GLM results).

\section{HF-HRV and General Self-Evaluated Health in Midlife}

Results from this model revealed no significant association between HF-HRV and selfevaluated health. This outcome was consistent at the univariate level, $\beta=.03, p=.31$, after 
controlling for previous self-evaluated health, $\beta=.04, p=.27$, and in the final model with the addition of covariates, $\beta=.05, p=.18(F D R q=.05)$.

\section{HF-HRV and Mortality}

Results from a binary logistic regression revealed a significant association between HFHRV and mortality at the univariate level, $B=-.36, S E=.11, p=.001, O R=.70,95 \% \mathrm{CI}[0.57$, 0.86]. The final model, presented in Table 5, which included all covariates, also revealed a significant association between HF-HRV and mortality, $B=-.23, S E=.11, p=.035$ (FDR $q=$ $.037), O R=.79,95 \% \mathrm{CI}[0.64,0.99]$, such that greater HF-HRV predicted decreased odds of mortality.

\section{Testing the Mediation Models}

Mediation analysis revealed a significant indirect effect of childhood parental warmth, through HF-HRV, on cardiovascular health, $B=-.02, S E=.01,95 \%$ CI [-.043, -.003], presented in Figure 1. Results from our robustness check, using sum of cardiovascular problems, again revealed a significant indirect effect of parental warmth, through HF-HRV, on sum of cardiovascular health problems, $B=-.01, S E=.005,95 \% \mathrm{CI}[-.022,-.002]$. Results from the mediation model predicting mortality revealed that the indirect effect of parental warmth, through HF-HRV, on mortality was not significant, $B=-.002,95 \%$ CI $[-.01, .00]$. The indirect effect of parental warmth, through HF-HRV, on self-evaluated health was also not significant, $B$ $=.11,95 \% \mathrm{CI}[-.002, .01]$.

\section{Post-Hoc Sensitivity Analysis}

Adjusted HF-HRV. Using HF-HRV adjusted for concurrent HR (aHF-HRV), controlling for the same covariates in the initial models, results were unchanged: parental warmth was positively associated with aHF-HRV $(B=.16, p=.014)$; higher aHF-HRV was 
associated with better cardiovascular health, using both a dichotomous variable $(B=-.17, p=$ $.006)$, and a count variable $(B=-.09, p=.004)$; aHF-HRV predicted reduced odds of mortality $(B=-.14, p=.014)$; and aHF-HRV did not significantly predict self evaluated health $(B=.02, p$ $=.48$ ). In addition, mediation results were unchanged, with aHF-HRV only significantly mediating the relation between parental warmth and cardiovascular health.

Moderation by Age. Due to the large age range of this sample, and the retrospective methods used for measuring childhood parental warmth, a sensitivity analysis was conducted to test age as a moderator of the relation between parental warmth and resting HF-HRV. Without covariates the interaction term between age and parental warmth was significant, $B=-.01, S E=$ $.005, p=.049$. A Johnson-Neyman region of significance test revealed that the relation between parental warmth and HF-HRV was only significant for individuals younger than 59 years old. After including covariates, the interaction term was no longer significant, $B=-.007, p=.17$.

\section{Discussion}

Parenting that is affectionate, consistent, and sensitive, or warm parenting, has often been associated with better physical health outcomes for offspring (Miller et al., 2011; Newland, 2015). A comprehensive understanding of the mechanisms involved in this association, including biological mechanisms, is still needed. The present study set out to investigate the associations between childhood parental warmth, resting high-frequency heart rate variability (HF-HRV), and health and mortality outcomes. Furthermore, we aimed to test whether ANS physiology may mediate the relation between parental warmth and health indices in older adulthood. Findings from the current study suggest that (1) retrospective measures of childhood parental warmth can predict resting HF-HRV in midlife, (2) resting HF-HRV can predict cardiovascular health over a nine year period, controlling for baseline cardiovascular health, and can also predict 
mortality, and (3) resting HF-HRV may act as a mediator between childhood parental warmth and health in older adulthood, though the indirect influence of parental warmth through HF-HRV on health may depend on the specific physical health outcomes measured.

Consistent with our first hypothesis, we found that individuals who reported greater parental warmth during childhood exhibited higher HF-HRV during rest. This is consistent with previous research on parental warmth and HRV with infants and adolescents (Graham et al., 2017; Porter, 2003). In general, these results shed light on the potential influence of parenting on ANS physiology (Propper \& Moore, 2006). Warm parenting may influence the offspring's lifelong autonomic physiology by providing stable, predictable environments that keep their physiological reactivity within certain set points (Flannery et al., 2017). This has important implications, as HF-HRV has been associated with several significant health outcomes (Thayer \& Lane, 2007).

The effect size was relatively small within our sample, compared to previous research in infants and adolescents (Graham et al., 2017; Porter, 2003). This may be attributed to a weakening association across development, as additional variables (e.g., health behavior, age) continue to influence ANS physiology into midlife. Alternatively, given the large sample used in the current study, this may reflect a true small effect. Nevertheless, a small effect of parental warmth on lifelong offspring physiology may have significant consequences at the population level and the individual level, particularly if it co-varies with early-life adversities, such as poverty. Results from our unadjusted model examining moderation by age provide some evidence that parental warmth may exhibit reduced influence on HF-HRV with age, though these results should be interpreted with caution as they were post-hoc and did not hold up to the inclusion of covariates. Alternatively, moderation by age results may reflect increasing memory 
bias with age, such that older individuals' retrospective reports may have had more measurement error. Further research using longitudinal designs and multiple measures of retrospective accounts of childhood experiences could help clarify these moderation results.

For the second aim of this study, we hypothesized that higher resting HF-HRV would predict better health outcomes, indexed through participants' reports of physician diagnosed cardiovascular health problems and self-evaluated health. Our findings were generally supportive of this hypothesis, and the effect was specific for cardiovascular health rather than a general effect on overall self-evaluated health. Specifically, we found that higher resting HF-HRV was associated with better cardiovascular health, indexed through (1) absence or presence of any diagnosed cardiovascular disorders, and (2) total number of cardiovascular health disorders. This association remained significant after statistically controlling for prior cardiovascular health, indicating that higher resting HF-HRV may protect against worsening in cardiovascular health over a 9-year period. These findings add to the large body of evidence suggesting an association between low resting HF-HRV and cardiovascular disease. While low resting HF-HRV has been associated with other health diagnoses, such as diabetes (Carnethon, Golden, Folsom, Haskell, \& Liao, 2003), the majority of past associations between low resting HF-HRV and physical health have focused on cardiovascular health, including: hypertension (Singh et al., 1998), high cholesterol (Christensen, Toft, Christensen, \& Schmidt, 1999), and coronary heart disease (Liao, Carnethon, Evans, Cascio, \& Heiss, 2002). It has been proposed that vagal activity has beneficial effects for cardiovascular health through multiple pathways, including a vagal anti-inflammatory pathway (Sloan et al., 2007; Tracey, 2007) that may reduce risk of atherosclerosis (Libby, 2002). Furthermore, low HF-HRV may also lead to poor health through its association with poor psychosocial functioning (Appelhans \& Luecken, 2006; Kemp \& Quintana, 2013). 
The large and nationally representative sample of adults and the longitudinal design of the MIDUS study provided a unique opportunity for also investigating the relation between HFHRV and mortality. Previous research on HRV and mortality suggests that higher resting HFHRV predicts reduced all-cause mortality risk (Thayer et al., 2010); the results were consistent with these past findings. While the association between HRV and cardiovascular disease may partially explain these findings, the all-cause mortality data included causes of death not directly related to cardiovascular health, such as cancer. However, considering the previously mentioned associations between low resting HRV and both (1) inflammatory processes (Tracey, 2007) and (2) poor psychosocial functioning (Kemp \& Quintana, 2013), it is possible that the mechanisms underlying the relation between low resting HF-HRV and cardiovascular disease are similar to those that drive the association between low resting HF-HRV and all-cause mortality.

The absence of a significant association between HF-HRV and global self-evaluated health suggests that HF-HRV may be more strongly related to specific health conditions, such as cardiovascular disease. It is possible that this global measure aggregates across many different aspects of health (e.g., mental health, physical limitations) that have weaker connections to highfrequency heart rate variability.

For the third aim of this study, we hypothesized that resting HF-HRV would mediate the relation between childhood parental warmth and health outcomes. Our findings were generally supportive of this, and the effect was specific for cardiovascular health rather than a general effect on overall self-reported health. Specifically, we found that childhood parental warmth was indirectly associated with better cardiovascular health, indexed through both absence of any diagnosed cardiovascular health disorders and fewer cardiovascular health disorders, and that this indirect association was explained through (1) the association between high childhood 
parental warmth and high resting HF-HRV, and (2) the association between high resting HFHRV and better cardiovascular health. These results are consistent with the model proposed by Repetti et al. (2002) whereby early-life family-environmental characteristics are theorized to affect offspring life-long health outcomes through their influence on offspring physiology. However, considering the weak indirect effect it is not likely that HF-HRV fully mediates the relation between parental warmth and cardiovascular health, and instead may work in addition to, or in an interaction with, other biological mediators (e.g., inflammation, the HPA axis).

While we did not observe a direct, bivariate relation between childhood parental warmth and cardiovascular health among this sample, contemporary mediation analysis no longer requires a direct association to be significant as a prerequisite to mediation analysis (MacKinnon, Krull, \& Lockwood, 2000; Shrout \& Bolger, 2002; Zhau, Lynch, \& Chen, 2010). Using simulation models, quantitative researchers have shown that mediation can occur even when an initial direct effect is not found (Hayes, 2009; Rucker, Preacher, Tormala, \& Petty, 2011). Such a phenomenon has been explained through (1) suppression, whereby unmeasured mediators express an indirect effect in the opposite direction, or (2) unbalanced statistical power, such as when the mediator has less measurement error than the independent or dependent variables, and therefore significant relations between either the independent or dependent variable and the mediator are easier to find than a significant relation between the independent and dependent variables (Rucker et al., 2011). Considering the expansive literature on the association between warmer parenting and better health (Chen, Brody, \& Miller, 2017), suppression is an unlikely explanation of our results. Instead, for the current study, the greater precision in the measurement of the biological mediator relative to the self-report methods employed for both the independent and dependent variables may have contributed to unbalanced statistical power, which allowed us 
to detect significant associations between parental warmth and HF-HRV, as well as HF-HRV and cardiovascular health outcomes, but not between self-reported parental warmth and cardiovascular health outcomes.

\section{Limitations and Future Directions}

While the current study addressed an important gap in the literature and has a number of strengths, there are some limitations that should be considered for future research. The first limitation is the use of a retrospective measure of parental warmth, which may be subject to memory bias (Hardt \& Rutter, 2004). However, due to the logistical constraints associated with longitudinal designs that span decades from childhood into middle/older age when chronic diseases begin to manifest, retrospective measurements are frequently utilized for investigating the long-term outcomes associated with parenting characteristics (Rothrauff, Cooney, \& An, 2009; Wright, Turner, \& McCarty, 2017). Stability of retrospective reports over time (Silva \& Maia, 2013) and modest but significant associations between observational and later retrospective measures of parenting (Newbury et al., 2018) provide some support for the validity of retrospective methods. In addition, the limitations associated with retrospective measures may be particularly important to consider within the current study due to the large age range, considering memory bias may increase with age. Concerns over this limitation may be partially alleviated by the inclusion of age as a covariate in our analyses.

Lastly, future research could benefit from employing an experimental design, for instance, by randomly assigning parents to receive parental warmth training; and from measuring HF-HRV at more than one time point, in order to directly observe change. The correlational design utilized in the current study limits our ability to interpret causality or direction of effects; it is possible that offspring autonomic physiology may influence parental warmth, as some have 
suggested (Hastings, Grady, \& Barrieau, 2018; Kennedy, Rubin, Hastings, \& Maisel, 2004), or that the relation is bidirectional. It is also possible that the association between childhood parental warmth and HF-HRV may be in part explained by shared genetics related to autonomic physiology. For example, it is possible that parents who themselves have high resting HF-HRV may be more likely to engage in warmer parenting, and pass on a genetic predisposition to high resting HF-HRV to their offspring. However, previous research has shown both intraindividual change in HRV across early years of development (Bornstein \& Suess, 2000), and discordant HRV among monozygotic twin pairs (Healy, 1992), suggesting substantial environmental contributions to HRV that need to be clarified.

Despite these limitations, the current study adds to the limited literature on the relation between parental warmth and ANS physiology, and is the first to investigate ANS physiology as a mediator between parental warmth, and health and mortality. Among a nationally representative sample of adults, we found that retrospective measures of childhood parental warmth were positively associated with resting HF-HRV, and that higher resting HF-HRV predicted better cardiovascular health and decreased all-cause mortality risk. We also found that HF-HRV may partially mediate the relation between parental warmth and health, and that this relation may be specific to cardiovascular health. A better understanding of the biological mechanisms behind the association between family-environmental factors and health contributes important evidence of potentially lasting beneficial implications of warm parenting, and provides unique opportunities for intervention, such as biofeedback training. 


\section{References}

Appelhans, B. M., \& Luecken, L. J. (2006). Heart rate variability as an index of regulated emotional responding. Review of General Psychology, 10(3), 229-240.

https://doi.org/10.1037/1089-2680.10.3.229

Atkins, D. C., \& Gallop, R. J. (2007). Rethinking how family researchers model infrequent outcomes: A tutorial on count regression and zero-inflated models. Journal of Family Psychology, 21(4), 726-735. doi:http://dx.doi.org/10.1037/0893-3200.21.4.726

Bell, Z., Shader, T., Webster-Stratton, C., Reid, M. J., \& Beauchaine, T. P. (2018). Improvements in negative parenting mediate changes in children's autonomic responding following a preschool intervention for ADHD. Clinical Psychological Science, 6(1), 134144. doi:http://dx.doi.org/10.1177/2167702617727559

Benjamins, M. R., Hummer, R. A., Eberstein, I. W., \& Nam, C. B. (2004). Self-reported health and adult mortality risk: An analysis of cause-specific mortality. Social Science \& Medicine, 59(6), 1297-1306. doi:http://dx.doi.org/10.1016/j.socscimed.2003.01.001

Bornstein, M. H., \& Suess, P. E. (2000). Physiological self-regulation and information processing in infancy: Cardiac vagal tone and habituation. Child Development, 71(2), 273287. https://doi.org/10.1111/1467-8624.00143

Calkins, S. D., Graziano, P. A., Berdan, L. E., Keane, S. P., \& Degnan, K. A. (2008). Predicting cardiac vagal regulation in early childhood from maternal-child relationship quality during toddlerhood. Developmental Psychobiology, 50(8), 751-766.

doi:http://dx.doi.org/10.1002/dev.20344

Carnethon, M. R., Golden, S. H., Folsom, A. R., Haskell, W., \& Liao, D. (2003). Prospective investigation of autonomic nervous system function and the development of type 2 diabetes: 
The atherosclerosis risk in communities study, 1987-1998. Circulation, 107(17), 21902195. https://doi.org/10.1161/01.CIR.0000066324.74807.95

Chen, E., Brody, G. H., \& Miller, G. E. (2017). Childhood close family relationships and health. American Psychologist, 72(6), 555-566. https://doi.org/10.1037/amp0000067

Chen, E., Miller, G. E., Kobor, M. S., \& Cole, S. W. (2011). Maternal warmth buffers the effects of low early-life socioeconomic status on pro-inflammatory signaling in adulthood. Molecular Psychiatry, 16(7), 729-737. doi:http://dx.doi.org/10.1038/mp.2010.53

Christensen, J., Toft, E., Christensen, M., \& Schmidt, E. (1999). Heart rate variability and plasma lipids in men with and without ischaemic heart disease. Atherosclerosis, 145, 181186.

Davids, E. L., Roman, N. V., \& Leach, L. (2017). The link between parenting approaches and health behavior: A systematic review. Journal of Human Behavior in the Social Environment, 27(6), 589-608. https://doi.org/10.1080/10911359.2017.1311816

de Geus, E. J. C., Gianaros, P. J., Brindle, R. C., Jennings, J. R., \& Berntson, G. G. (2019). Should heart rate variability be "corrected" for heart rate? Biological, quantitative, and interpretive considerations. Psychophysiology, 56(2), 1-26. https://doi.org/10.1111/psyp.13287

Donoho, C. J., Seeman, T. E., Sloan, R. P., \& Crimmins, E. M. (2015). Marital Status, Marital Quality, and Heart Rate Variability in the MIDUS Cohort. Journal of Family Psychology, 29(2), 290-295. https://doi.org/10.1037/fam0000068.

Farrell, A. K., Simpson, J. A., Carlson, E. A., Englund, M. M., \& Sung, S. (2016). The impact of stress at different life stages on physical health and the buffering effects of maternal sensitivity. Health Psychology, 36(1), 35-44. https://doi.org/10.1037/hea0000424 
Flannery, J. E., Beauchamp, K. G., \& Fisher, P. A. (2017). The role of social buffering on chronic disruptions in quality of care: Evidence from caregiver-based interventions in foster children. Social Neuroscience, 12(1), 86-91. doi:http://dx.doi.org/10.1080/17470919.2016.1170725

Fox, A. R., Aldrich, J. T., Ahles, J. J., \& Mezulis, A. H. (2019). Stress and parenting predict changes in adolescent respiratory sinus arrhythmia. Developmental Psychobiology, 00, 111. doi:http://dx.doi.org/10.1002/dev.21863

Fuller-Rowell, T., Curtis, D. S., Chae, D. H., \& Ryff, C. D. (2018). Longitudinal health consequences of socioeconomic disadvantage: Examining perceived discrimination as a mediator. Health Psychology, 37(5), 491-500. doi:http://dx.doi.org/10.1037/hea0000616

Gerritsen, J., Dekker, J. M., Tenvoorde, B. J., Kostense, P. J., Heine, R. J., Bouter, L. M., ... Stehouwer, C. D. A. (2001). Impaired autonomic function is associated with increased mortality, especially in subjects with diabetes, hypertension, or a history of cardiovascular disease: The Hoorn study. Diabetes Care, 24(10), 1793-1798. https://doi.org/10.2337/diacare.24.10.1793

Graham, R. A., Scott, B. G., \& Weems, C. F. (2017). Parenting behaviors, parent heart rate variability, and their associations with adolescent heart rate variability. Journal of Youth and Adolescence, 46(5), 1089-1103. https://doi.org/10.1007/s10964-016-0616-x

Graves, P. L., Wang, N. Y., Mead, L. A., Johnson, J. V., \& Klag, M. J. (1998). Youthful precursors of midlife social support. Journal of Personality and Social Psychology, 74(5), 1329-1336. https://doi.org/10.1037/0022-3514.74.5.1329

Gunnar, M. R., \& Quevedo, K. (2007). The neurobiology of stress and development. Annual Review of Psychology, 58, 145-173. doi: 10.1146/annurev.psych.58.110405.085605 
Hardt, J., \& Rutter, M. (2004). Validity of adult retrospective reports of adverse childhood experiences: Review of the evidence. Journal of Child Psychology and Psychiatry and Allied Disciplines, 45(2), 260-273. https://doi.org/10.1111/j.1469-7610.2004.00218.x

Hastings, P., Grady, J., \& Barrieau, L. (2018). Children's Anxious Characteristics Predict how their Parents Socialize Emotions. Journal of Abnormal Child Psychology. https://doi.org/10.1007/s10802-018-0481-z

Hastings, P. D., Kahle, S., Fleming, C., Lohr, M. J., Katz, L. F., \& Oxford, M. L. (2018). An intervention that increases parental sensitivity in families referred to child protective services also changes toddlers' parasympathetic regulation. Developmental Science, 22:e12725. doi:http://dx.doi.org/10.1111/desc.12725

Hayes, A. F. (2009). Beyond Baron and Kenny: Statistical Mediation Analysis in the New Millennium. Communication Monographs. https://doi.org/10.1080/03637750903310360

Healy, B. T. (1992). The heritability of autonomic nervous system processes. In T. M. Field, P. M. McC- abe, \& N. Schneiderman (Eds.), Stress and coping in infancy and childhood (pp. 69-82). Hillsdale, NJ: Erlbaum.

Hostinar, C. E., Sullivan, R. M., \& Gunnar, M. R. (2014). Psychobiological mechanisms underlying the social buffering of the hypothalamic-pituitary-adrenocortical axis: A review of animal models and human studies across development. Psychological Bulletin. 140(1), 256-282. https://doi.org/10.1037/a0032671

Kemp, A. H., \& Quintana, D. S. (2013). The relationship between mental and physical health: Insights from the study of heart rate variability. International Journal of Psychophysiology, 89(3), 288-296. https://doi.org/10.1016/j.ijpsycho.2013.06.018

Kennedy, A. E., Rubin, K. H., Hastings, P. D., \& Maisel, B. (2004). Longitudinal relations 
between child vagal tone and parenting behavior: 2 to 4 years. Developmental Psychobiology, 45(1), 10-21. https://doi.org/10.1002/dev.20013

Kluttig, A., Kuss, O., \& Greiser, K. H. (2010). Ignoring lack of association of heart rate variability with cardiovascular disease and risk factors: Response to the manuscript "the relationship of autonomic imbalance, heart rate variability cardiovascular disease risk factors" by Julian F. Thayer, Shelby S. Yamamoto, Jos F. Brosschot. International Journal of Cardiology, 145(2), 375-376. https://doi.org/10.1016/j.ijcard.2010.02.048

Laborde, S., Mosley, E., \& Thayer, J. F. (2017). Heart rate variability and cardiac vagal tone in psychophysiological research - Recommendations for experiment planning, data analysis, and data reporting. Frontiers in Psychology, 8(FEB), 1-18. https://doi.org/10.3389/fpsyg.2017.00213

Liao, D., Cai, J., Brancati, F. L., Folsom, A., Barnes, R. W., Tyroler, H. A., \& Heiss, G. (1995). Association of vagal tone with serum insulin, glucose, and diabetes mellitus - The ARIC Study. Diabetes Research and Clinical Practice, 30(3), 211-221. https://doi.org/10.1016/S0369-8114(01)00239-5

Liao, D., Carnethon, M., Evans, G. W., Cascio, W. E., \& Heiss, G. (2002). Lower heart rate variability is associated with the development of coronary heart disease in individuals. Diabetes, 51(December), 3524-3531. https://doi.org/10.2337/diabetes.51.12.3524

Libby, P. (2002). Inflammation in atherosclerosis. Nature, 420, 868. Retrieved from https://doi.org/10.1038/nature01323

MacKinnon, D. P., Krull, J. L., \& Lockwood, C. M. (2000). Equivalence of the mediation, confounding, and suppression effect. Prevention Science, 1, 173-181.

Malik, M. (1996). Heart rate variability. Standards of measurement, physiological interpretation, 
and clinical use. Task Force of the European Society of Cardiology and the North American Society of Pacing and Electrophysiology. Eur. Heart J. 17, 354-381.

Miller, G. E., \& Chen, E. (2010). Harsh family climate in early life presages the emergence of a proinflammatory phenotype in adolescence. Psychological Science, 21(6), 848-856. doi:http://dx.doi.org/10.1177/0956797610370161

Miller, G. E., Chen, E., \& Parker, K. J. (2011). Psychological stress in childhood and susceptibility to the chronic diseases of aging: Moving toward a model of behavioral and biological mechanisms. Psychological Bulletin, 137(6), 959-997. doi:http://dx.doi.org/10.1037/a0024768

Morris, A. S., Silk, J. S., Steinberg, L., Myers, S. S., \& Robinson, L. R. (2007). The role of the family context in the development of emotion regulation. Social Development, 16(2), 361388. https://doi.org/10.1111/j.1467-9507.2007.00389.x

Newbury, J. B., Arseneault, L., Moffitt, T. E., Caspi, A., Danese, A., Baldwin, J. R., \& Fisher, H. L. (2018). Measuring childhood maltreatment to predict early-adult psychopathology: Comparison of prospective informant-reports and retrospective self-reports. Journal of Psychiatric Research, 96, 57-64. doi: 10.1016/j.jpsychires.2017.09.020

Newland, L. A. (2015). Family well-being, parenting, and child well-being: Pathways to healthy adjustment. Clinical Psychologist, 19(1), 3-14. https://doi.org/10.1111/cp.12059

Nozdrachev, A. D., \& Shcherbatykh, Y. V. (2001). Modern methods of functional studies of the autonomic nervous system. Human Physiology, 27(6), 732-737. doi:http://dx.doi.org/10.1023/A:1012993430373

Perry, N. B., Nelson, J. A., Swingler, M. M., Leerkes, E. M., Calkins, S. D., Marcovitch, S., \& O'Brien, M. (2013). The relation between maternal emotional support and child 
physiological regulation across the preschool years. Developmental Psychobiology, 55(4), 382-394. doi:http://dx.doi.org/10.1002/dev.21042

Porges, S. W. (2007). The polyvagal perspective. Biological Psychology, 74(2), 116-143. https://doi.org/10.1016/j.biopsycho.2006.06.009

Porter, C. L. (2003). Coregulation in mother-infant dyads: Links to infants' cardiac vagal tone. Psychological Reports, 92(1), 307-319. Doi:http://dx.doi.org/10.2466/PR0.92.1.307319

Propper, C., \& Moore, G. A. (2006). The influence of parenting on infant emotionality: A multilevel psychobiological perspective. Developmental Review, 26(4), 427-460. https://doi.org/10.1016/j.dr.2006.06.003

Repetti, R. L., Taylor, S. E., \& Seeman, T. E. (2002). Risky families: Family social environments and the mental and physical health of offspring. Psychological Bulletin, 128(2), 330-366. https://doi.org/10.1037//0033-2909.128.2.330

Rossi, A. (2001). Developmental roots of adult social responsibility. In A. S. Rossi (Ed.), Caring and doing for others: Social responsibility in the domains of family, work, and community (pp. 227-320). Chicago, IL: University of Chicago Press.

Rothrauff, T. C., Cooney, T. M., \& An, S. J. (2009). Remembered parenting styles and adjustment in middle and late adulthood. Journals of Gerontology - Series B Psychological Sciences and Social Sciences, 64(1), 137-146. https://doi.org/10.1093/geronb/gbn008

Rucker, D. D., Preacher, K. J., Tormala, Z. L., \& Petty, R. E. (2011). Mediation Analysis in Social Psychology: Current Practices and New Recommendations. Social and Personality Psychology Compass. https://doi.org/10.1111/j.1751-9004.2011.00355.x

Shrout, P. E., \& Bolger, N. (2002). Mediation in experimental and nonexperimental studies: New 
procedures and recommendations. Psychological Methods, 7, 422-445.

Silva, S., \& Maia, A., (2013). The stability of self-reported adverse experiences in childhood: A longitudinal study on obesity. Journal of Interpersonal Violence, 28(10), 1989-2004. doi:http://dx.doi.org/10.1177/0886260512471077

Singh, J.P., Larson, M.G., Tsuji, H., Evans, J.C., O’Donnell, C.J., \& Levy, D., (1998). Reduced heart rate variability and new-onset hypertension. Insights into pathogenesis of hypertension: the Framington heart study. Hypertension 32, 293-297.

Sloan, R. P., McCreath, H., Tracey, K. J., Sidney, S., Liu, K., \& Seeman, T. (2007). RR interval variability is inversely related to inflammatory markers: the CARDIA study. Molecular medicine (Cambridge, Mass.), 13(3-4), 178-84.

Sloan, R. P., Schwarz, E., McKinley, P. S., Weinstein, M., Love, G., Ryff, C., .. Seeman, T. (2017). Vagally-mediated heart rate variability and indices of well-being: Results of a nationally representative study. Health Psychology, 36(1), 73-81. doi:http://dx.doi.org/10.1037/hea0000397

Tabachnick, A. R., Raby, K. L., Goldstein, A., Zajac, L., \& Dozier, M. (2019). Effects of an attachment-based intervention in infancy on children's autonomic regulation during middle childhood. Biological Psychology, 143, 22-31. doi:http://dx.doi.org/10.1016/j.biopsycho.2019.01.006

Thayer, J. F., \& Lane, R. D. (2007). The role of vagal function in the risk for cardiovascular disease and mortality. Biological Psychology, 74(2), 224-242. https://doi.org/10.1016/j.biopsycho.2005.11.013

Thayer, J. F., Yamamoto, S. S., \& Brosschot, J. F. (2010). The relationship of autonomic imbalance, heart rate variability and cardiovascular disease risk factors. International 
Journal of Cardiology, 141(2), 122-131. https://doi.org/10.1016/j.ijcard.2009.09.543

Thompson, R. A. (1994). Emotion regulation: A theme in search of definition. Monographs of the Society for Research in Child Development, 59(2-3), 25-52, 250-283. doi:http://dx.doi.org/10.2307/1166137

Tracey, K. J. (2007). Physiology and immunology of the cholinergic antiinflammatory pathway. Journal of Clinical Investigation. https://doi.org/10.1172/JCI30555

Tsuji, H., Venditti, F. J., Manders, E. S., Evans, J. C., Larson, M. G., Feldman, C. L., \& Levy, D. (1994). Reduced heart rate variability and mortality risk in an elderly cohort. The Framingham Heart Study. Circulation, 90(2), 878-883.

https://doi.org/10.1161/01.CIR.90.2.878

Uchino, B. N., \& Way, B. M. (2017). Integrative pathways linking close family ties to health: A neurochemical perspective. American Psychologist, 72(6), 590-600. https://doi.org/10.1037/amp0000049

Wiley, J. F., Gruenewald, T. L., Karlamangla, A. S., \& Seeman, T. E. (2016). Modeling multisystem physiological dysregulation. Psychosomatic Medicine, 78(3), 290-301. https://doi.org/10.1097/PSY.0000000000000288

Wright, C. N., Turner, L. A., \& McCarty, D. L. (2017). Perceived parenting, self-conscious emotions, and forgiveness in college students. Counseling and Values, 62(1), 57-71. https://doi.org/10.1002/cvj.12049

Zhao, X., Lynch, J. G., \& Chen, Q. (2010). Reconsidering Baron and Kenny: Myths and Truths about Mediation Analysis. Journal of Consumer Research. https://doi.org/10.1086/651257 Zhou, Q., Eisenberg, N., Losoya, S. H., Fabes, R. A., Reiser, M., Guthrie, I. K., . . Shepard, S. A. (2002). The relations of parental warmth and positive expressiveness to children's 
empathy-related responding and social functioning: A longitudinal study. Child

Development, 73(3), 893-915. doi:http://dx.doi.org/10.1111/1467-8624.00446 


\section{Tables}

Table 1. Major Variable Descriptives and Sample Characteristics

\begin{tabular}{|c|c|c|c|c|}
\hline Variable & $N$ & $M$ & $S D$ & Range \\
\hline HF-HRV & 1,148 & 4.92 & 1.28 & $0.90-9.66$ \\
\hline Heart Rate & 1,148 & 72.84 & 10.85 & $44.4-109.8$ \\
\hline aHF-HRV & 1,148 & 0.97 & 1.45 & $-3.84-5.95$ \\
\hline Parental Warmth & 1,223 & 2.92 & 0.66 & $0.96-3.96$ \\
\hline Number of CV Problems & 938 & 0.79 & 0.90 & $0-5$ \\
\hline Number of CV Problems (MIDUS 2) & 1,227 & 0.79 & 0.97 & $0-6$ \\
\hline Self-Evaluated Health ${ }^{\mathrm{a}}$ & 943 & 2.54 & 1.02 & $0-4$ \\
\hline Self-Evaluated Health ${ }^{\mathrm{b}}$ (MIDUS 2) & 1,010 & 7.84 & 3.08 & $0-10$ \\
\hline Age (years) & 1,255 & 57.32 & 11.55 & $35-86$ \\
\hline \multirow[t]{2}{*}{ Childhood SES ${ }^{\mathrm{c}}$} & 1,217 & 5.82 & 2.86 & $1-12$ \\
\hline & $N$ & Level & Frequency & Percent \\
\hline \multirow[t]{2}{*}{ Cardiovascular Problems } & 938 & At least one & 524 & 55.9 \\
\hline & & None & 414 & 44.1 \\
\hline \multirow[t]{2}{*}{ Cardiovascular Problems (MIDUS 2) } & 1,244 & At least one & 647 & 52.0 \\
\hline & & None & 597 & 48.0 \\
\hline \multirow[t]{2}{*}{ Deceased } & 1,255 & Yes & 84 & 6.7 \\
\hline & & No & 1171 & 93.3 \\
\hline \multirow[t]{2}{*}{ Sex } & 1,255 & Female & 713 & 56.8 \\
\hline & & Male & 542 & 43.2 \\
\hline \multirow[t]{5}{*}{ Race } & 1,251 & Caucasian & 985 & 78.5 \\
\hline & & African American & 215 & 17.2 \\
\hline & & Native American & 17 & 1.4 \\
\hline & & Asian & 3 & 0.2 \\
\hline & & Other & 31 & 2.5 \\
\hline Engages in Regular Exercise & 1,255 & Yes & 960 & 76.5 \\
\hline Current Smoker & 1,255 & Yes & 187 & 14.9 \\
\hline Medication that Increases HF-HRV & 1,255 & Yes & 186 & 14.8 \\
\hline Medication that Decreases HF-HRV & 1,255 & Yes & 195 & 15.5 \\
\hline Menopause Status & 1,255 & Post & 248 & 19.8 \\
\hline
\end{tabular}

Note. $\mathrm{HF}-\mathrm{HRV}=$ high-frequency heart rate variability. aHF-HRV $=\mathrm{HF}-\mathrm{HRV}$ adjusted for heart rate. $\mathrm{CV}=$ cardiovascular. ${ }^{\text {a }}$ Self-evaluated Health during MIDUS 3 ranged from: $0=$ poor to $4=$ excellent. ${ }^{\mathrm{b}}$ Self-Evaluated Health during MIDUS 2 ranged from: $0=$ worst to $10=$ best. ${ }^{\mathrm{c}}$ Childhood SES was indexed through highest parental education level, and ranged from: $1=$ no school/some grade school to $12=$ doctoral degree. 


\section{HEART RATE VARIABILITY IN MIDLIFE}

Table 2. Bivariate Correlations Between Study Variables and Covariates

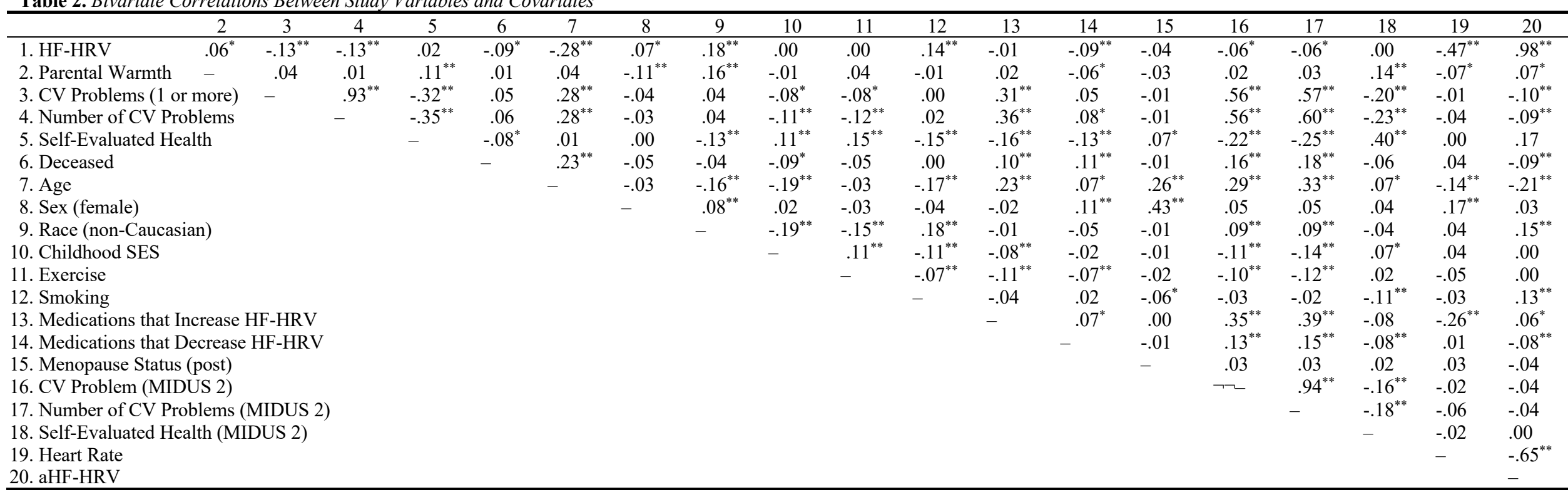

Note. HF-HRV = high frequency-heart rate variability. $\mathrm{CV}=$ cardiovascular. aHF-HRV = HF-HRV adjusted for heart rate. Correlations calculated using Spearman's rho.

${ }^{*} p<.05,{ }^{* *} p<.01$. 
HEART RATE VARIABILITY IN MIDLIFE

Table 3. Multiple Linear Regression Predicting Resting HF-HRV

\begin{tabular}{lrrrrrc}
\hline & \multicolumn{3}{c}{ Step 1 } & \multicolumn{3}{c}{ Step 2 } \\
\cline { 2 - 7 } \multicolumn{1}{c}{ Predictor } & $B$ & $S E$ & $\beta$ & $B$ & $S E$ & $\beta$ \\
\hline \multicolumn{1}{c}{ Constant } & 4.53 & .18 & & 6.04 & .30 \\
Parental Warmth & .12 & .06 & $.06^{*}$ & .14 & .06 & $.07^{*}$ \\
Age & & & & -.03 & .004 & $-.27^{* *}$ \\
Sex (female) & & .17 & .08 & $.06^{*}$ \\
Race (non-Caucasian) & & .34 & .10 & $.11^{* *}$ \\
Childhood SES & & -.01 & .01 & -.02 \\
Exercise & & .04 & .09 & .01 \\
Smoking & .25 & .11 & $.07^{*}$ \\
Medication that Increases HF-HRV & .25 & .11 & $.07^{*}$ \\
Medication that Decreases HF-HRV & -.28 & .10 & $-.08^{* *}$ \\
Menopause Status & .01 & .11 & .002 \\
\hline
\end{tabular}

Note. HF-HRV = high-frequency heart rate variability.

$* p<.05, * * p<.01$. 
HEART RATE VARIABILITY IN MIDLIFE

Table 4. Generalized Linear Model Predicting Total Sum of Cardiovascular Health Problems

\begin{tabular}{|c|c|c|c|c|c|c|c|c|c|}
\hline \multirow[b]{2}{*}{ Predictor } & \multicolumn{3}{|c|}{ Step 1} & \multicolumn{3}{|c|}{ Step 2} & \multicolumn{3}{|c|}{ Step 3} \\
\hline & $B$ & $S E$ & $95 \% \mathrm{CI}$ & $B$ & $S E$ & $95 \% \mathrm{CI}$ & $B$ & $S E$ & $95 \% \mathrm{CI}$ \\
\hline Constant & 0.28 & .16 & & -0.28 & .17 & & 0.28 & .43 & \\
\hline HF-HRV & $-.12 * *$ & .03 & {$[-.19,-.06]$} & $-.10 * *$ & .03 & {$[-.17,-.04]$} & $-.10 * *$ & .04 & {$[-.17,-.03]$} \\
\hline \multicolumn{3}{|c|}{ CV Problems (MIDUS 2) } & & $.52 * *$ & .03 & {$[.45, .59]$} & $.44 * *$ & .04 & {$[.36, .52]$} \\
\hline \multicolumn{3}{|l|}{ Age } & & & & & .01 & .004 & {$[-.002, .01]$} \\
\hline \multicolumn{3}{|l|}{ Sex (female) } & & & & & .08 & .09 & {$[-.11, .26]$} \\
\hline \multicolumn{3}{|c|}{ Race (non-Caucasian) } & & & & & -.27 & .14 & {$[-.55, .01]$} \\
\hline \multicolumn{3}{|l|}{ Childhood SES } & & & & & -.01 & .02 & {$[-.04, .02]$} \\
\hline \multicolumn{3}{|l|}{ Exercise } & & & & & .10 & .10 & {$[-.09, .29]$} \\
\hline \multicolumn{3}{|l|}{ Smoking } & & & & & -.20 & .12 & {$[-.45, .04]$} \\
\hline \multicolumn{4}{|c|}{ Medication that Increases HF-HRV } & & & & $-.45 * *$ & .10 & {$[-.65,-.25]$} \\
\hline \multicolumn{4}{|c|}{ Medication that Decreases HF-HRV } & & & & -.05 & .11 & {$[-.27, .16]$} \\
\hline \multicolumn{4}{|c|}{ Menopause Status } & & & & -.02 & .12 & {$[-.25, .21]$} \\
\hline
\end{tabular}

Note. HF-HRV = high-frequency heart rate variability. CV = cardiovascular. 95\% CI = [lower limit, upper limit $]$ of 95\% Wald confidence interval of $B$.

$* p<.05, * * p<.01$. 
Table 5. Binary Logistic Regression Predicting Mortality by the MIDUS 3 assessment

\begin{tabular}{|c|c|c|c|c|c|c|}
\hline \multirow[b]{2}{*}{ Predictor } & \multicolumn{3}{|c|}{ Step 1} & \multicolumn{3}{|c|}{ Step 2} \\
\hline & $B$ & $S E$ & OR & $B$ & $S E$ & $O R$ \\
\hline Constant & -1.20 & .48 & & -6.36 & 1.24 & \\
\hline HF-HRV & $-.36 * *$ & .11 & .70 & $-.23 *$ & .11 & .79 \\
\hline Age & & & & $.08 * *$ & .01 & 1.08 \\
\hline Sex (female) & & & & -.53 & .34 & .59 \\
\hline Race (non-Caucasian) & & & & .44 & .38 & 1.55 \\
\hline Childhood SES & & & & -.04 & .06 & .96 \\
\hline Exercise & & & & -.11 & .32 & .90 \\
\hline Smoking & & & & .54 & .41 & 1.71 \\
\hline Medication that Increas & F-HRV & & & .43 & .33 & 1.54 \\
\hline Medication that Decrea & IF-HRV & & & $1.06^{* *}$ & .32 & 2.88 \\
\hline Menopause Status & & & & .05 & .43 & 1.05 \\
\hline
\end{tabular}

Note. HF-HRV $=$ high-frequency heart rate variability. $O R=$ odds ratio.

$* p<.05, * * p<.01$. 
HEART RATE VARIABILITY IN MIDLIFE

\section{Figure Captions}

Figure 1. Model of resting high-frequency heart rate variability (HF-HRV) as a mediator between parental warmth and presence of a cardiovascular health disorder.

$* p<.05 . * * p<.01$ 


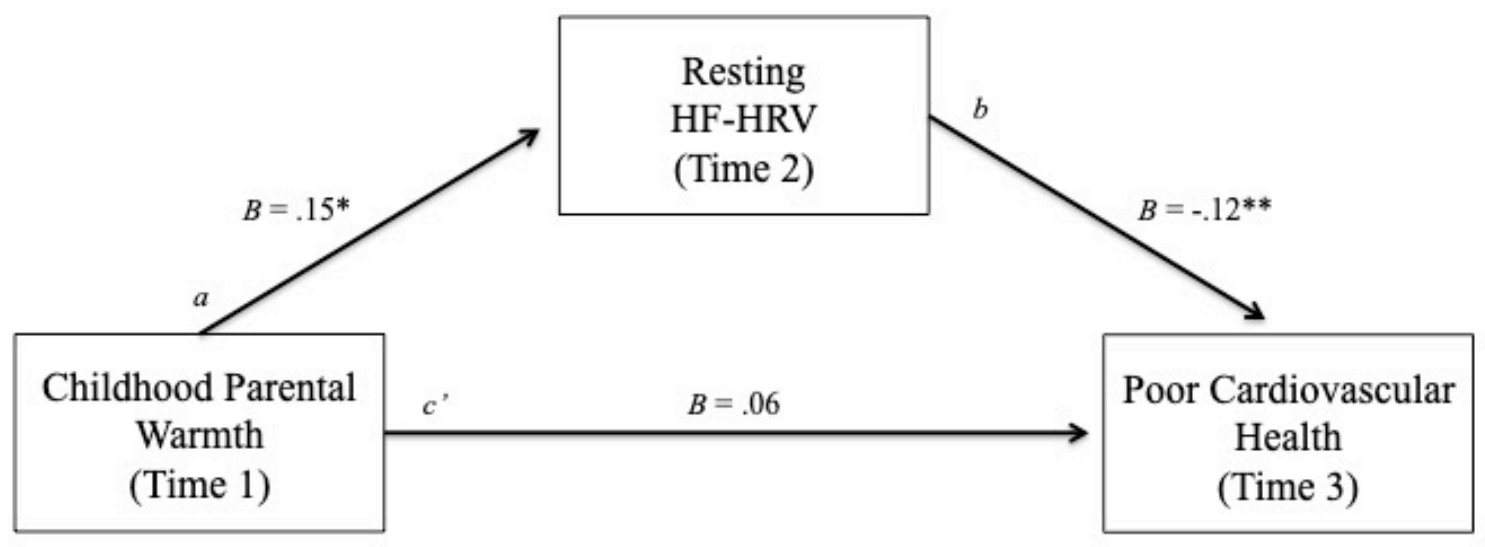

Total effect $(c): B=.04, S E=.08, p=.58$

Indirect effect $\left(a^{*} b\right): B=-.02, S E=.01,95 \%$ Bootstrapped CI $[-.043,-.003]$ 